\title{
Imaging the water snow-line during a protostellar outburst
}

Lucas A. Cieza ${ }^{1,2}$, Simon Casassus ${ }^{2,3}$, John Tobin ${ }^{4}$, Steven P. Bos ${ }^{4}$, Jonathan P. Williams ${ }^{5}$, Sebastian Perez², , Zhaohuan Zhu $^{6}$, Claudio Caceres ${ }^{2,7}$, Hector Canovas ${ }^{2,7}$, Michael M. Dunham ${ }^{8}$, Antonio Hales ${ }^{9}$, Jose L. Prieto ${ }^{1,10}$, David A. Principe ${ }^{1,2}$, Matthias R. Schreiber ${ }^{2,7}$, Dary Ruiz-Rodriguez ${ }^{11}$ \& Alice Zurlo ${ }^{1,2,3}$

A snow-line is the region of a protoplanetary disk at which a major volatile, such as water or carbon monoxide, reaches its condensation temperature. Snow-lines play a crucial role in disk evolution by promoting the rapid growth of ice-covered grains ${ }^{1-6}$. Signatures of the carbon monoxide snow-line (at temperatures of around 20 kelvin) have recently been imaged in the disks surrounding the pre-main-sequence stars TW Hydra ${ }^{7-9}$ and HD163296 (refs 3, 10), at distances of about 30 astronomical units (AU) from the star. But the water snow-line of a protoplanetary disk (at temperatures of more than 100 kelvin) has not hitherto been seen, as it generally lies very close to the star (less than 5 AU away for solar-type stars ${ }^{11}$ ). Water-ice is important because it regulates the efficiency of dust and planetesimal coagulation ${ }^{5}$, and the formation of comets, ice giants and the cores of gas giants ${ }^{12}$. Here we report images at 0.03 -arcsec resolution (12 $\mathrm{AU})$ of the protoplanetary disk around V883 Ori, a protostar of 1.3 solar masses that is undergoing an outburst in luminosity arising from a temporary increase in the accretion rate $^{13}$. We find an intensity break corresponding to an abrupt change in the optical depth at about $42 \mathrm{AU}$, where the elevated disk temperature approaches the condensation point of water, from which we conclude that the outburst has moved the water snow-line. The spectral behaviour across the snow-line confirms recent model predictions ${ }^{14}$ : dust fragmentation and the inhibition of grain growth at higher temperatures results in soaring grain number densities and optical depths. As most planetary systems are expected to experience outbursts caused by accretion during their formation ${ }^{15,16}$, our results imply that highly dynamical water snow-lines must be considered when developing models of disk evolution and planet formation.

V883 Ori is an FU Orionis (FU Ori) type star that was identified as such ${ }^{17}$ via follow-up spectroscopy of deeply embedded sources from the Infrared Astronomical Satellite. It is located in the Orion Nebula Cluster, which is at a distance of $414 \pm 7$ parsecs from Earth ${ }^{18}$. The mass of V883 Ori's protoplanetary disk is greater than about $0.3 M_{\odot}$ (where $M_{\odot}$ is the mass of the Sun), and its bolometric luminosity is $400 L_{\odot}$ (ref. 19). We have obtained $230 \mathrm{GHz} / 1.3 \mathrm{~mm}$ (band-6) observations of V883 Ori using the Atacama Large Millimeter/Submillimeter Array (ALMA), in four different array configurations with baselines ranging from 14 metres to 12.6 kilometres, taken in ALMA cycles 2 and 3. These new ALMA observations include continuum and the ${ }^{12} \mathrm{CO},{ }^{13} \mathrm{CO}$, and $\mathrm{C}^{18} \mathrm{O} J=2-1$ spectral lines. We use the $\mathrm{C}^{18} \mathrm{O}$ gas line to investigate the dynamics of the system at $0.2^{\prime \prime}(90 \mathrm{AU})$ resolution, and the continuum data to constrain the physical properties of the dust in the V883 Ori disk at $0.03^{\prime \prime}(12 \mathrm{AU})$ resolution. In Fig. 1a we show our cycle- 3 continuum image at $0.03^{\prime \prime}$ resolution-the highest resolution ever obtained for a FU Ori object at millimetre wavelengths. We find that the V883 Ori disk has a two-region morphology, with a very bright inner disk (radius $\sim 0.1^{\prime \prime}, 42 \mathrm{AU}$ ) and a much more tenuous outer disk extending
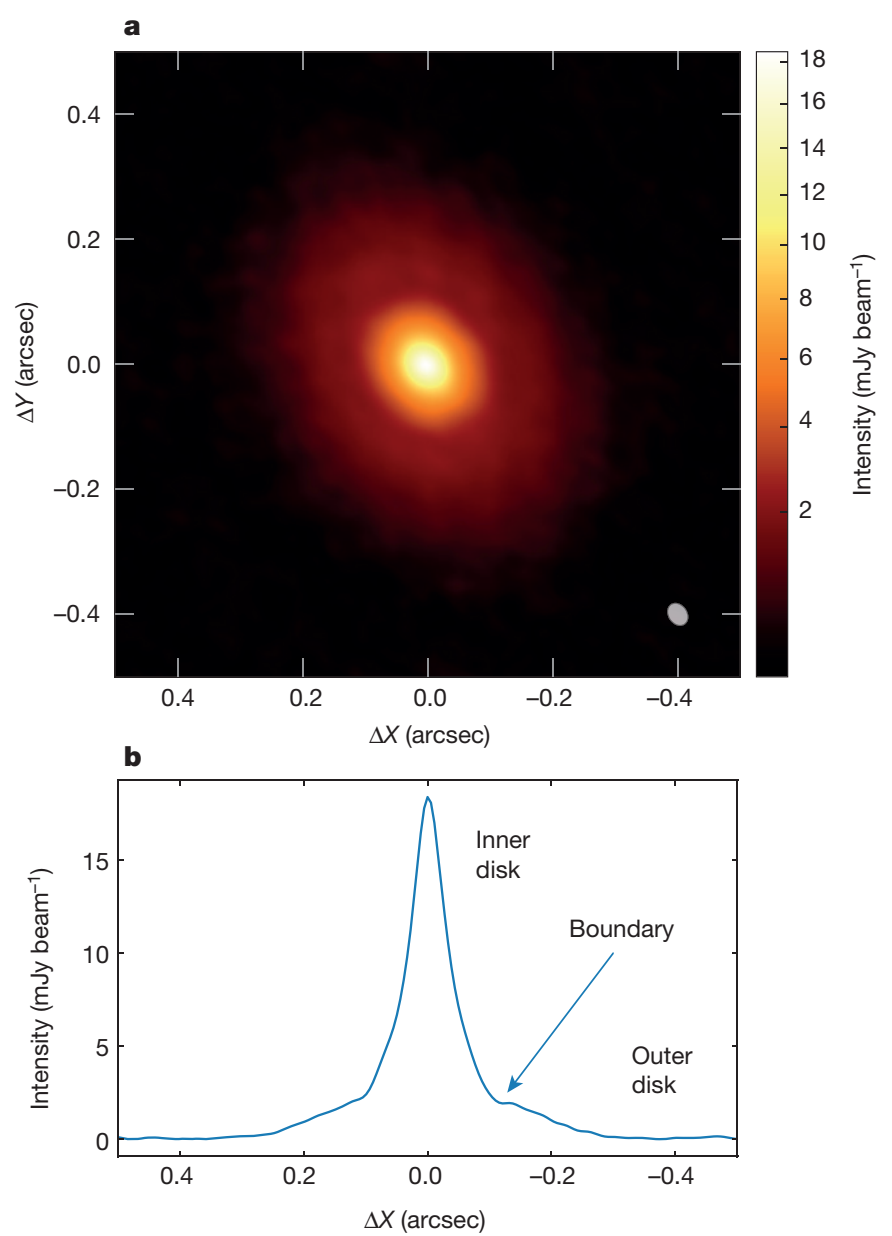

Figure 1 | ALMA observations of V883 Ori. a, The band- 6 image at $0.03^{\prime \prime}(12 \mathrm{AU})$ resolution obtained on 27 October 2015. b. The intensity profile along the major axis. There is a very bright inner disk with radius $\sim 0.1^{\prime \prime}$ ( $42 \mathrm{AU}$ ), surrounded by a much more tenuous outer disk extending out to radius $\sim 0.3^{\prime \prime}(125 \mathrm{AU})$. The boundary between these two regions is sharp and probably unresolved. $X$ and $Y$ are the right ascension and the declination, respectively. 

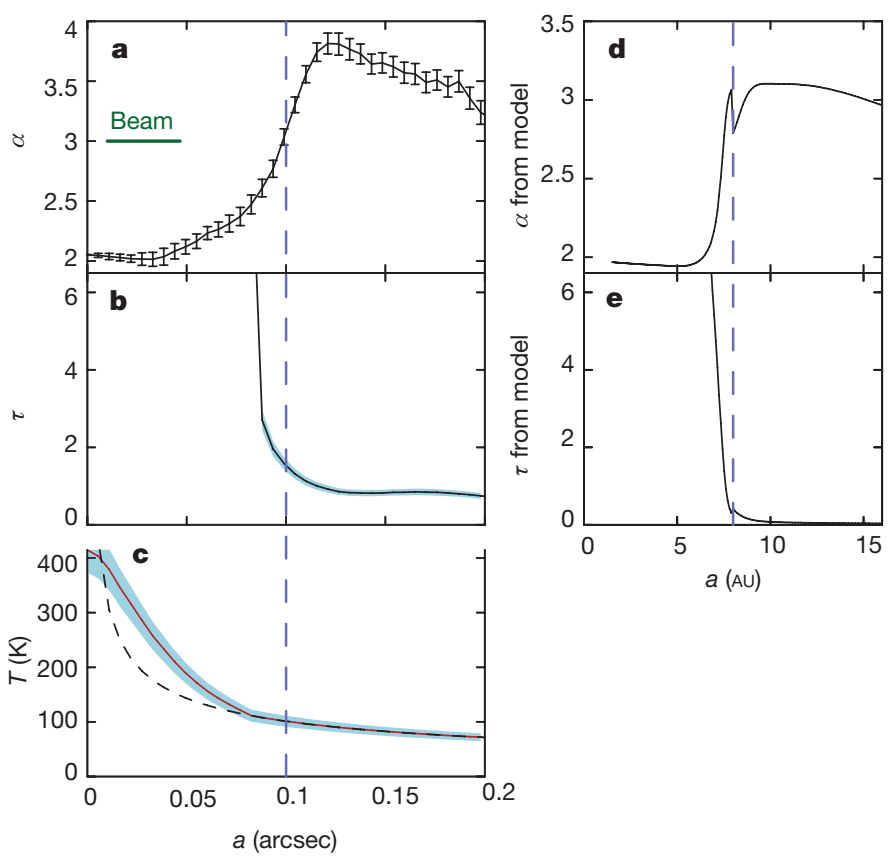

Figure 2 | Comparison of observations to models. a-c, The spectral index $(\alpha)$, the optical depth $(\tau)$, and the temperature $(T)$ that we derived from the observed data, all as a function of the semi-major axis, $a$. The temperature profile, shown in red in c, is fixed to a square-root law (shown by the black dashed line) at the radii at which $\tau$ drops below 3 . The uncertainties (error bars and light blue regions) are 68\% confidence intervals (see Methods). The blue dashed line corresponds to the location of the water snow-line. $\alpha$ and $\tau$ are dimensionless. d, e, Predictions from the model in ref. 14 for a disk viscosity of $10^{-4}$ and an optically thick inner disk.

out to $\sim 0.3^{\prime \prime}$ (125 AU). The brightness profile (Fig. 1b) indicates that what looks like a ring at $0.1^{\prime \prime}$ in the continuum image is really a sharp transition between these two regions.

Our continuum observations include two different spectral windows, each $1.875 \mathrm{GHz}$ wide and centred at $218.0 \mathrm{GHz}$ and $232.6 \mathrm{GHz}$, respectively (see Methods). Even though these spectral windows are separated by only $14.6 \mathrm{GHz}$, the very high signal-to-noise ratio of our observations allows us to derive accurate information regarding the spectral behaviour of the spatially resolved disk emission out to a radius of about $0.2^{\prime \prime}(85 \mathrm{AU})$. We use concentric ellipses (inclination $=38.3^{\circ}$ and position angle $=32.4^{\circ}$ ) to extract radial profiles in each spectral window as a function of the semi-major axis, $a$. We compare the radial profiles extracted in the two spectral windows separately. In order to have perfectly matched UV coverage in the two spectral windows, we degraded the resolution of the $232.6 \mathrm{GHz}$ observations to match that of the $218.0 \mathrm{GHz}$ data. The spectral index, $\alpha=\ln \left(F_{232.6 \mathrm{GHz}} / F_{218.0 \mathrm{GHz}}\right) /$ $\ln (218.0 \mathrm{GHz} / 232.6 \mathrm{GHz})$, as a function of $a$ is shown in Fig. $2 \mathrm{a}(F$ is the flux at the frequency shown). We find distinct spectral behaviours across the disk, with $\alpha=2.02 \pm 0.03$ in the central beam of the inner disk (corresponding to optically thick black-body emission, with temperatures of more than $100 \mathrm{~K}$ ), and $\alpha$ reaching $3.7 \pm 0.2$ in the outer disk (typical of optically thin interstellar-medium values).

The observed spectral trends can be cast in terms of physical conditions, with grey-body fits ${ }^{20}$ that can be used as diagnostics for the optical depth, $\tau(a)=\tau_{0} \times\left(\nu / \nu_{0}\right)^{\beta}$, and for the average dust temperature along the line of sight $\left(T_{\mathrm{s}}\right.$, summed to $\tau \approx 1$ ) (where $\nu$ is the frequency of the emission and $\beta$ is the power-law index relating $\tau$ and $\nu$ ). In our case, the spectral information available is an amplitude and a slope, at the reference frequency $v_{0}=218.0 \mathrm{GHz}$. Because we are provided with only two data points, we fix $\beta=1.0$, as appropriate for circumstellar material $^{21}$. The inner disk is very optically thick, and we can obtain an accurate estimate for $T_{\mathrm{s}}$ that is independent of the adopted $\beta$. On the other hand, $\tau_{0}$ and $T_{\mathrm{s}}$ become degenerate in the optically thin regime.
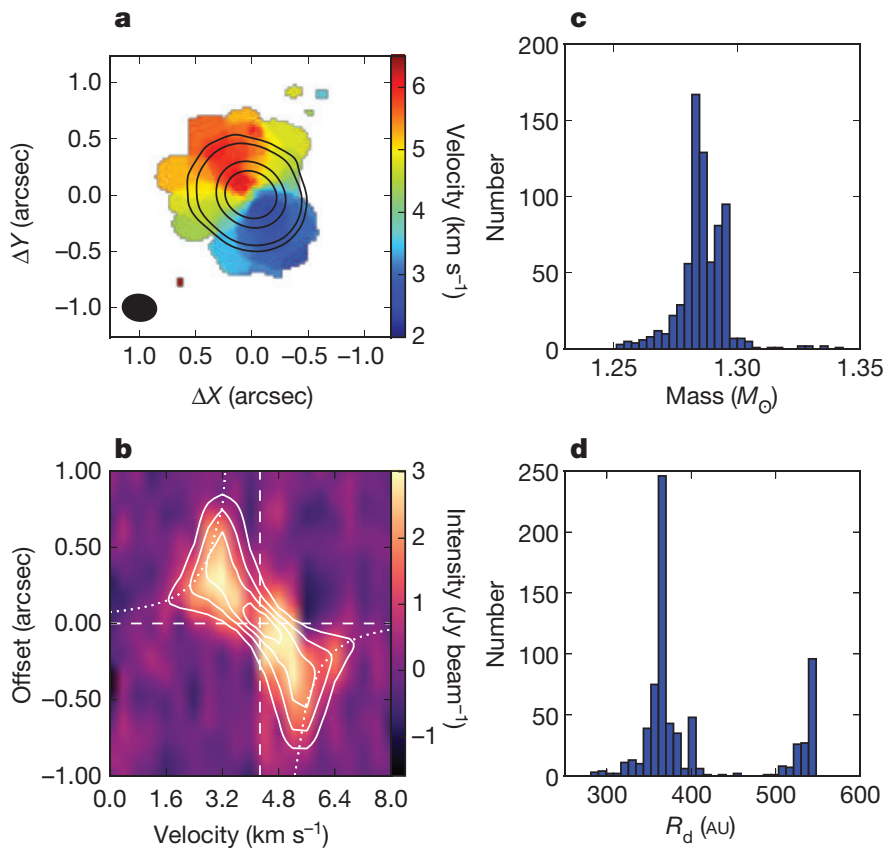

Figure 3 | Dynamical mass estimate. a, Intensity-weighted mean velocity map of the $\mathrm{C}^{18} \mathrm{O}$ line, revealing clear Keplerian rotation. Continuum flux contours have been superposed. $X$ and $Y$ are the right ascension and the declination, respectively. $\mathbf{b}$, Position-velocity diagram of the $\mathrm{C}^{18} \mathrm{O} J=2-1$ line. The horizontal and vertical dashed lines denote the position of the source and the source velocity. The curved dotted lines correspond to a Keplerian rotation curve, assuming a central mass of $1.3 M_{\odot}$. c, d, Distribution of $\mathbf{c}$, the masses of the central object, and d, the radii of the disk $\left(R_{\mathrm{d}}\right)$, resulting from the minimum $\chi^{2}$ fittings.

We therefore adopt a temperature profile with $T_{\mathrm{s}} \propto 1 / \sqrt{a}$ extrapolated from the region where $\tau=3$. The corresponding $\tau$ and $T_{\mathrm{s}}$ profiles for these assumptions are shown in Fig. 2b, c. We find that the sharp (unresolved) break at $\sim 0.1^{\prime \prime}$ seen in the V883 Ori disk (Fig. 1a) is associated with a steep drop in optical depth and with the transition from the optically thick to the optically thin regime. This result is robust and insensitive to $\beta$ and to the exact prescription used to estimate $\tau$ and $T_{\mathrm{s}}$ beyond $0.1^{\prime \prime}$.

This intensity break occurs where the temperature has dropped below $105 \pm 11 \mathrm{~K}$. This temperature is more consistent with a water snow-line than with the snow-line of any of the other major volatiles present in protoplanetary disks (carbon monoxide, carbon dioxide and methane). The sublimation temperature of water is a strong function of ambient pressure. While it can be close to $\sim 100 \mathrm{~K}$ in the interstellar medium, high-vacuum laboratory experiments and simulations ${ }^{22-24}$ suggest that it should be $\sim 150-170 \mathrm{~K}$ at the $10^{-4}$ bar pressures expected at the location of the water snow-line in a typical disk $(1-5 \mathrm{AU})^{11,25}$. However, because the pressure is lower at $\sim 40 \mathrm{AU}$, the sublimation temperature should also be lower in the case of V883 Ori. Furthermore, our temperature estimate is based on an extrapolation from the surface of the optically thick inner disk and might underestimate the true temperature of the water snowline owing to the intense viscous heating at the disk midplane ${ }^{25}$.

The observed spectral behaviour across the water snow-line has recently been predicted ${ }^{14}$ from numerical models that include the radial drift, coagulation, and fragmentation of dust grains. In Fig. 2 we show predictions for these models based on low disk viscosity $\left(\alpha_{\mathrm{vis}}=10^{-4}\right)$, which result in an optically thick inner disk, as appropriate for V883 Ori. The model predictions are not convolved with the ALMA beam and thus have higher resolution than our observations. In these models, the fragmentation velocity of dust is 1 metre per second inside the snow-line and 10 metres per second for ice-covered grains outward of this line. In this scenario, icy grains quickly grow into centimetre-sized pebbles. Some of these icy particles drift into the inner disk, where their icy mantles evaporate. When this happens, their drift velocity 
decreases, while the fragmentation efficiency increases. This produces an accumulation of millimetre-sized grains in the inner disk, driving the $230 \mathrm{GHz}$ opacity up and the spectral index to the optically thick limit of $\sim 2$. In these models ${ }^{14}, \alpha$ increases and $\tau$ decreases steeply around the water snow-line, in remarkably good agreement with our observational results. Our ALMA observations thus represent both a confirmation of the predictions of ref. 14 and the first resolved image of the signatures of the water snow-line in a protoplanetary disk.

By fitting a Keplerian model to the $\mathrm{C}^{18} \mathrm{O}$ line data, we derive a dynamical mass of $(1.3 \pm 0.1) M_{\odot}$ for the central source (see Fig. 3 and Methods). Assuming an age of 0.5 million years (Myr), as appropriate for a class I protostar such as V883 Ori (ref. 15), its photospheric luminosity should be a mere $\sim 6 L_{\odot}$ (ref. 26). On the basis of the stellar mass and the observed luminosity of $400 L_{\odot}$ (ref. 19), we derive an accretion rate of $7 \times 10^{-5} M_{\odot}$ per year, which is typical of FU Ori objects ${ }^{13}$. The location of the water snow-line in a protoplanetary disk is mostly determined by accretion heating in young Solar-type stars ${ }^{11,25}$. For a $1 M_{\odot}$ star, the snow-line begins at $\sim 5 \mathrm{AU}$ at disk formation and moves inward to $\sim 1$ AU by an age of a few million years, driven by the steady decrease in the accretion rate during disk evolution ${ }^{11}$. However, as shown by V883 Ori, this steady evolution is punctuated by extreme bursts of accretion that can drive the snow-line out to more than $40 \mathrm{AU}$.

In contrast to the HL Tau protoplanetary disk ${ }^{27}$, whose concentric gaps have been interpreted as revealing the occurrence of planet formation at condensation fronts ${ }^{1}$, the optical depth structure in V883 Ori is close to a step-function, as would be expected for efficient grain growth beyond a critical radius. Outward of the water snow-line, grains are covered by ice and can coagulate more efficiently into snowballs and eventually icy planetesimals ${ }^{28}$. Inside the snow-line, on the other hand, ice mantles evaporate, increasing the efficiency of destructive collisions and resulting in the production of a new population of small dust grains ${ }^{29}$. In this scenario, illustrated in Extended Data Fig. 1, an FU Ori outburst can increase the optical depth at millimetre wavelengths of a large region of the disk, by melting snowballs and releasing silicate grains from their icy mantles, in turn triggering further dust production. If the HL Tau ring system is in fact due to planet formation promoted by the condensation fronts, then the case of V883 Ori would represent an even earlier stage of disk evolution. Substantial evolution of solids (their growth, migration and fragmentation) has already occurred, but dynamical clearing of gaps by a planet has not yet happened.

While the fact that V883 Ori might reveal some of the very early steps towards planet formation is fascinating in itself, the outward movement of the water snow-line during FU Ori outbursts has far-reaching consequences for our understanding of disk evolution and planet formation in general. The water snow-line establishes the basic architecture of planetary systems like our own: in our Solar System, rocky planets formed inward of this line in the protosolar nebula, while giants formed outside. However, the intimate relation between the position of the water snow-line and the evolution of the central star is not yet understood. Although present population-synthesis models for planets do consider a steady decrease in the accretion rate during disk evolution and the corresponding inward motion of the water snow-line at the planet-formation epoch ${ }^{11,25}$, they do not take into account the dramatic effects that FU Ori outbursts have on the snow-line location during the class I stage. If most systems experience FU-Ori-type outbursts during their evolution, as proposed in the episodic accretion scenario ${ }^{13,15,16}$, then this implies that highly dynamical snow-lines must be taken into consideration in planet-formation models.

Online Content Methods, along with any additional Extended Data display items and Source Data, are available in the online version of the paper; references unique to these sections appear only in the online paper.

Received 15 March; accepted 18 May 2016.

1. Zhang, K., Blake, G. A. \& Bergin, E. A. Evidence of fast pebble growth near condensation fronts in the HL Tau protoplanetary disk. Astrophys. J. $\mathbf{8 0 6}$ L7-L12 (2015).
2. Okuzumi, S., Tanaka, H., Kobayashi, H. \& Wada, K. Rapid coagulation of porous dust aggregates outside the snow line: a pathway to successful icy planetesimal formation. Astrophys. J. 752, 106-123 (2012).

3. Guidi, G. et al. Dust properties across the CO snowline in the HD 163296 disk from ALMA and VLA observations. Astron. Astrophys. 588, A112-A123 (2016).

4. Baillié, K., Charnoz, S. \& Pantin, E. Time evolution of snow regions and planet traps in an evolving protoplanetary disk. Astron. Astrophys. 577, A65-A76 (2015).

5. Blum, J. \& Wurm, G. The growth mechanisms of macroscopic bodies in protoplanetary disks. Annu. Rev. Astron. Astrophys. 46, 21-56 (2008).

6. Zhang, K. et al. On the commonality of 10-30 au sized axisymmetric dust structures in protoplanetary disks. Astrophys. J. 818, L16-L22 (2016).

7. Qi, C. et al. Imaging of the CO snow line in a solar nebula analog. Science $\mathbf{3 4 1 ,}$ 630-632 (2013).

8. Nomura, $\mathrm{H}$. et al. ALMA observations of a gap and a ring in the protoplanetary disk around TW Hya. Astrophys. J. 819, L7-L13 (2016)

9. Schwarz, K. et al. The radial distribution of $\mathrm{H}_{2}$ and $\mathrm{CO}$ in TW Hya as revealed by resolved ALMA observations of CO isotopologues. Astrophys. J. 823, $91 \mathrm{~S}$ (2016).

10. Qi, C. et al. Chemical imaging of the CO snow line in the HD 163296 disk. Astrophys. J. 813, 128 (2015).

11. Kennedy, G. \& Kenyon, S. Planet formation around stars of various masses: the snow line and the frequency of giant planets. Astrophys. J. 673, 502-512 (2008).

12. Morbidelli, A., Lambrechts, M., Jacobson, S. \& Bitsch, B. The great dichotomy of the Solar System: small terrestrial embryos and massive giant planet cores. Icarus 258, 418-429 (2015).

13. Audard, M. et al. in Protostars and Planets VI (eds Beuther, H., Klessen, R. S., Dullemond, C. P. \& Henning, T.) 387-410 (Univ. Arizona Press, 2014)

14. Banzatti, A. et al. Direct imaging of the water snow line at the time of planet formation using two ALMA continuum bands. Astrophys. J. 815, L15-L20 (2015).

15. Evans, N. et al. The Spitzer c2d legacy results: star-formation fates and efficiencies; evolution and lifetimes. Astrophys. J. 181 (Suppl.), 321-350 (2009).

16. Dunham, M. \& Vorobyov, E. Resolving the luminosity problem in low-mass star formation. Astrophys. J. 747, 52-72 (2012).

17. Strom, K. \& Strom, S. The discovery of two FU Orionis objects in L1641. Astrophys. J. 412, L63-L66 (1993).

18. Menten, K. M., Reid, M. J., Forbrich, J. \& Brunthaler, A. The distance to the Orion Nebula. Astron. Astrophys. 474, 515-520 (2007)

19. Sandell, G. \& Weintraub, D. On the similarity of FU Orionis stars to class protostars: evidence from the submillimeter. Astrophys. J. 134 (Suppl.), 115-132 (2001)

20. Casassus, S. et al. A compact concentration of large grains in the HD 142527 protoplanetary dust trap. Astrophys. J. 812, 126-139 (2015).

21. Williams, J. \& Cieza, L. Protoplanetary disks and their evolution. Annu. Rev. Astron. Astrophys. 49,67-117 (2011).

22. Collings, M. et al. A laboratory survey of the thermal desorption of astrophysically relevant molecules. Mon. Not. R. Astron. Soc. 354, 1133-1140 (2004).

23. Fayolle, E. et al. Laboratory $\mathrm{H}_{2} \mathrm{O}: \mathrm{CO}_{2}$ ice desorption data: entrapment dependencies and its parameterization with an extended three-phase model. Astron. Astrophys. 529, A74-A84 (2011).

24. Martín-Doménech, R., Muñoz Caro, G. M., Bueno, J. \& Goesmann, F. Thermal desorption of circumstellar and cometary ice analogs. Astron. Astrophys. 564, A8-A19 (2014)

25. Mulders, G., Ciesla, F., Min, M. \& Pascucci, I. The snow line in viscous disks around low-mass stars: implications for water delivery to terrestrial planets in the habitable zone. Astrophys. J. 807, 9-15 (2015)

26. Siess, L. Dufour, E \& Forestini, M. An internet server for pre-main sequence tracks of low- and intermediate-mass stars. Astron. Astrophys. 358, 593-599 (2000).

27. ALMA Partnership et al. The 2014 ALMA long baseline campaign: first results from high angular resolution observations toward the HL Tau region. Astrophys. J. 808, L3-L12 (2015).

28. Ros, K. \& Johansen, A. Ice condensation as a planet formation mechanism. Astron. Astrophys. 552, A137-A150 (2013).

29. Birnstiel, T., Dullemond, C. P. \& Brauer, F. Gas- and dust evolution in protoplanetary disks. Astron. Astrophys. 513, A79-A99 (2010).

Acknowledgements We thank the referees for their valuable comments. We also thank A. Banzatti and P. Pinilla for providing their model predictions in tabular form (Fig. 2d, e). ALMA is a partnership of the European Southern Observatory (ESO; representing its member states), the National Science Foundation (NSF; USA) and the National Institutes of Natural Sciences (Japan), together with the National Research Council (Canada) and the National Science Council and the Academia Sinica Institute of Astronomy and Astrophysics (Taiwan), in cooperation with the Republic of Chile. The Joint ALMA Observatory is operated by ESO, Associated Universities Inc./National Radio Astronomy Observatory (NRAO), and the National Astronomical Observatory of Japan. The NRAO is a facility of the NSF, operated under cooperative agreement by Associated Universities. Support for this work was provided by the Millennium Science Initiative (Chilean Ministry of Economy), through grants RC130007 and IC120009. L.A.C., D.A.P., J.L.P. and C.C. acknowledge support from CONICYT 


\section{LETTER RESEARCH}

FONDECYT grants $1140109,3150550,1151445$ and 3140592, respectively. H.C. acknowledges support from the Spanish Ministerio de Economía y Competitividad under grant AYA2014-55840P. Our work made use of ALMA data available at https://almascience.eso.org/alma-data with the following accession numbers: 2013.1.00710.S and 2015.1.00350.S

Author Contributions L.A.C. led the ALMA cycle-2 and cycle-3 proposals (with the contribution of most of the other authors) and the writing of the manuscript. S.C. analysed the cycle-3 data and performed the grey-body analysis. J.T. and S.A.B. determined the stellar dynamical mass. J.P.W. analysed the cycle-2 molecular line data. S.P. and Z.Z. performed the simulations supporting the cycle-3 proposal. All co-authors commented on the manuscript and contributed to the interpretation of the results.

Author Information Reprints and permissions information is available at www.nature.com/reprints. The authors declare no competing financial interests. Readers are welcome to comment on the online version of the paper. Correspondence and requests for materials should be addressed to L.A.C. (lucas.cieza@mail.udp.cl).

Reviewer Information Nature thanks $\mathrm{E}$. Bergin and the other anonymous reviewer(s) for their contribution to the peer review of this work. 


\section{METHODS}

Our band-6 cycle-2 observations were taken under ALMA program 2013.1.00710.S with three different antenna configurations. V883 Ori was observed on 12 December 2014 and 5 April 2015, using 37 and 39 antennas on the C34-2/1 and C34-1/2 configurations, respectively. The minimum and maximum baselines of both configurations are very similar: minimum $\sim 14$ metres and maximum $\sim 350$ metres. The integration time was $\sim 2$ minutes in each epoch. The target was re-observed on 30 August 2015, with 35 antennas on the C34-7/6 configuration and with baselines in the range 42 metres to 1.5 kilometres. In this configuration, the integration time was $\sim 3$ minutes. For all antenna configurations, the ALMA correlator was configured so that three spectral windows with $58.6 \mathrm{MHz}$ bandwidths were centred at $230.5380 \mathrm{GHz}, 220.3987 \mathrm{GHz}$ and $219.5603 \mathrm{GHz}$, to cover the ${ }^{12} \mathrm{CO} J=2-1,{ }^{13} \mathrm{CO} J=2-1$ and $\mathrm{C}^{18} \mathrm{O} J=2-1$ transitions, respectively. Two additional spectral windows with $1.875 \mathrm{GHz}$ bandwidths were centred at 232.6 GHz and $218.0 \mathrm{GHz}$ for continuum observations. The moon Ganymede and the quasar J0423-013 were used as flux calibrators, while the quasars J0538-4405 and J0607-0834 were observed for bandpass calibration. Observations of nearby phase calibrators (the quasars J0541 - 0541, J0532-0307 and/or J0529-0519) were alternated with our present target to calibrate the time-dependence variations of the complex gains.

V883 Ori was also observed in cycle-3 under program 2015.1.00350.S on 27 October 2015, with 45 antennas in the C38-8 configuration. This is the most extended array configuration offered in cycle- 3 , with baselines ranging from 267 metres to 12.6 kilometres. The total on-source integration time was 23 minutes. The correlator set-up was identical to that for our cycle-2 observations. J05410541 and J0529-0519 were used as primary and secondary phase calibrators, respectively. J0423-0120 was observed as a bandpass calibrator, and also as the primary flux calibrator. All data were calibrated using the Common Astronomy Software Applications package (CASA v4.4.0) ${ }^{30}$ by the ALMA observatory. The standard calibration included offline water vapour radiometer (WVR) calibration, system temperature correction, and bandpass, phase and amplitude calibrations. Continuum images and spectral-line data cubes were created from the pipelinecalibrated visibilities using the CLEAN routine and Briggs weighting in the CASA v4.4.0 software package. Continuum subtraction was performed in the visibility domain before imaging the CO lines. Similarly, CLEANing of the dust continuum was performed after removing channels containing line emission.

All of the cycle-2 observations (three epochs with three different array configurations) were combined together to produce a single $\mathrm{C}^{18} \mathrm{O}$ data cube. The root mean squared (r.m.s.) in this data cube is $10 \mathrm{mJy}_{\text {beam }}{ }^{-1}$ per $0.25 \mathrm{~km} \mathrm{~s}^{-1}$ channel, with a beam of size $\sim 0.35^{\prime \prime}$ by $\sim 0.27^{\prime \prime}$ and a position angle of $89.9^{\circ}$. The long-baseline cycle- 3 data set was reduced by itself in a similar fashion to the cycle- 2 observations. The continuum data resulted in a $0.029^{\prime \prime} \times 0.038^{\prime \prime}$ beam, and a r.m.s. of $0.05 \mathrm{mJy}$ beam $^{-1}$, after one iteration of phase-only self-calibration.

The grey-body diagnostics used as a proxy for physical conditions of the dust require comparable UV coverages in both continuum frequencies. However, the difference in frequency in simultaneous observations implies a corresponding radial shift in the UV coverage. We followed two independent approaches to build such comparable maps, and confirmed that the two approaches provide very similar spectral-index maps and physical conditions. We first obtained two restored maps with multiscale Cotton-Schwabb CLEAN ${ }^{31}$ by splitting off each spectral window, with the same CLEAN masks. We then degraded the higher frequency with an elliptical Gaussian whose axes correspond to the difference in quadrature of both clean beams. We also followed a second method, based on non-parametric Bayesian image synthesis. We fit an image model to the observations at each spectral window, and in the visibility domain, by minimizing the weighted least-square distance, as previously done in other multifrequency analyses of ALMA data ${ }^{20,32}$. Because both approaches provided very similar trends, we adopted the Bayesian image synthesis, as it potentially allows for slightly finer angular detail, and the residual were more homogeneous (that is, free from structure) across the image than were the CLEAN residuals. Following ref. 20, we performed grey-body fits to our 218.0 GHz and $232.6 \mathrm{GHz}$ images, such that $I_{\nu}(a)=B_{\nu}\left(T_{\mathrm{s}}(a)\right)[1-\exp (\tau(a))]$, where the optical depth is calculated as $\tau(a)=\tau_{0} \times\left(\nu / \nu_{0}\right)^{\beta}, I_{\nu}$ is the specific intensity, $B_{\nu}$ is the spectral radiance of the grey body, and $T_{s}$ is the average dust temperature along the line of sight (summed to $\tau \sim 1$ ). In our case, the spectral information available is an amplitude and a slope, at the reference frequency $v_{0}=218.0 \mathrm{GHz}$. We fixed $\beta=1.0$, as appropriate for circumstellar material ${ }^{14}$. We estimated the error bars (68\% confidence intervals) on the spectral indices from the r.m.s. scatter of specific intensities within each elliptical bin, summed in quadrature with the r.m.s. intensity of the image-synthesis residuals. The uncertainties in the $\tau$ and temperature profiles (light blue regions in Fig. 2b, c) are given by a systematic flux calibration error of $10 \%$ ( $68 \%$ confidence interval).

Molecular-line kinematics around a central object are most commonly analysed by creating a position-velocity diagram. The $\mathrm{C}^{18} \mathrm{O}$ line signal was too weak to be detected at the high spatial resolution of the cycle- 3 observations; therefore, we use the cycle- 2 data at $0.2^{\prime \prime}$ resolution to investigate the gas dynamics. Figure 3 shows the flux as a function of the velocity and position along the major axis of the disk. In this diagram, the position is shown as an offset from the centre of the disk, and the flux has been integrated along the width of the cut $\left(1.5^{\prime \prime}\right.$ in the direction of the semi-major axis). The disk position angle was set to $32.4^{\circ} \pm 2^{\circ}$, determined from an elliptical Gaussian fit. The diagram shows separated blue-shifted and red-shifted components, suggesting Keplerian rotation. The radius of the disk visible in the diagram is $0.75^{\prime \prime}$, or about $320 \mathrm{AU}$. The central part around the source velocity $\left(4.3 \mathrm{~km} \mathrm{~s}^{-1}\right)$ traces the outer slowly rotating material, and is largely resolved out owing to the extended emission. We also see that the data trace the higher velocities to $1.6 \mathrm{~km} \mathrm{~s}^{-1}$ and $6.6 \mathrm{~km} \mathrm{~s}^{-1}$.

To further analyse the $\mathrm{C}^{18} \mathrm{O}$ line emission and to give a mass estimate for the central object, we fitted a geometrically thin disk model, based on the model in ref. 33. On the basis of the channel maps and the position-velocity diagram, we decided to model a pure Keplerian disk without any infall. The velocity structure is then given by:

$$
v_{\phi}(r)=\sqrt{\frac{G M}{r}}
$$

where $v_{\phi}$ is the velocity of the gas in the disk at location $r, G$ is the gravitational constant, and $M$ is the mass of the central protostar. We conducted a $\chi^{2}$-minimization fitting using the method of ref. 34 :

$$
\chi^{2}=\frac{1}{N} \sum_{i=1}^{N}\left(\frac{D_{i}-M_{i}}{\sigma_{\text {r.m.s. }}}\right)^{2}
$$

where $D_{i}$ refers to the data, $M_{i}$ the model and $\sigma_{\text {r.m.s. }}$ the observed noise error observed for every velocity channel map. As this method will not search through the entire parameter space, we carried out multiple runs using different random starting parameters. The parameters used by the fitting algorithm were: the mass of the central object $(M)$, the radius of the Keplerian disk $\left(R_{\mathrm{d}}\right)$, the peak intensity $\left(I_{0}\right)$ and the full-width at half-maximum (FWHM) of the Gaussian intensity distribution. The position angle was again set to $32.4^{\circ}$, and we determined an inclination of $38.3 \pm 1^{\circ}$ from the ratio of the major and minor axes of the disk in the continuum image. We adopted a distance of 414 parsecs (ref. 18), and set the source velocity $\left(v_{\mathrm{src}}\right)$ to $4.3 \mathrm{~km} \mathrm{~s}^{-1}$ on the basis of the moment- 1 map. We fixed the centre of the disk to the centre of the continuum image. Of all these runs, the best fit (lowest $\left.\chi^{2}\right)$ gave the following parameters: $M=(1.29 \pm 0.02) M_{\odot}, R_{\mathrm{d}}=361 \pm 27 \mathrm{AU}$, $I_{0}=0.18 \pm 0.04 \mathrm{Jy} \mathrm{beam}^{-1}$ and FWHM $=1.14^{\prime \prime} \pm 0.11^{\prime \prime}$. The distributions of the parameters $M$ and $R_{\mathrm{d}}$ are shown in Fig. 3. A mass of around $1.3 M_{\odot}$ is the preferred solution. We adopt a $10 \%$ total error ( $68 \%$ confidence interval) in the dynamical mass to account for the uncertainty in the distance to V883 Ori, which dominates the error budget. There is more degeneracy in the size of the Keplerian disk, with values mainly varying between $300 \mathrm{AU}$ and $550 \mathrm{AU}$. Using the parameters given by the best fit, we overlaid contours of the model over the $\mathrm{C}^{18} \mathrm{O}$ emission as shown in Fig. 3. The shapes of the contours follow the data well. We see that the model traces higher velocities, which is to be expected as the model does not suffer from noise. A Keplerian rotation curve assuming a central mass of $1.3 M_{\odot}$ is also plotted in Fig. 3 . Most of the model and $\mathrm{C}^{18} \mathrm{O}$ emission fall well within this curve.

30. McMullin, J. P. et al. CASA architecture and applications. Astron. Soc. Pac. Conf. Ser. 376, 127-130 (2007).

31. Rau, U. \& Cornwell, T. A multi-scale multi-frequency deconvolution algorithm for synthesis imaging in radio interferometry. Astron. Astrophys. 532, A71-A87 (2011)

32. Casassus, S. et al. Flows of gas through a protoplanetary gap. Nature $\mathbf{4 9 3}$, 191-194 (2013).

33. Maret, S. Thindisk 1.0: compute the line emission from a geometrically thin protoplanetary disk. Zenodo https://zenodo.org/record/13823 (2015)

34. Powell, M. J. An efficient method for finding the minimum of a function of several variables without calculating derivatives. Computer J. 7, 155-162 (1964) 


\section{(a) Quiescent snow-line}

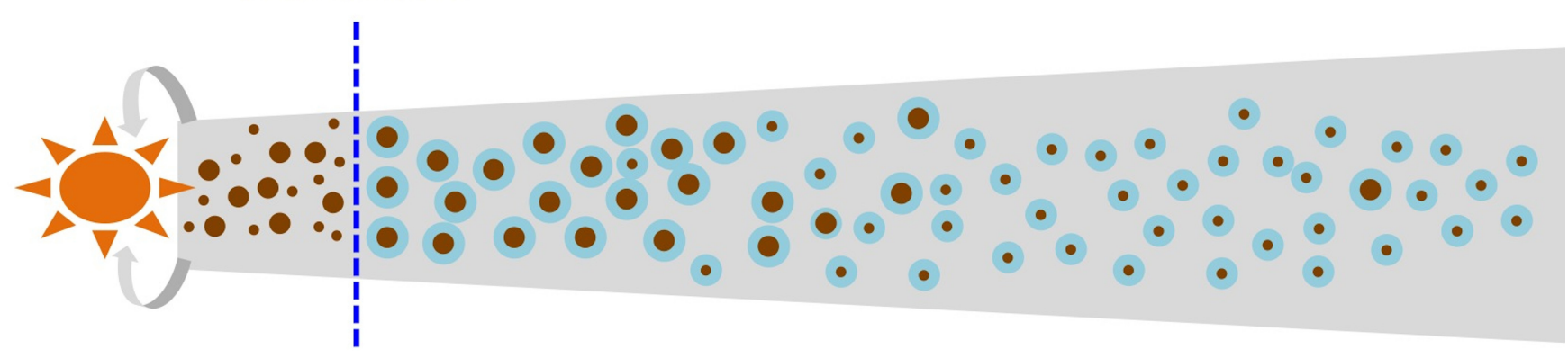

(b)

\section{Outburst}

\section{snow-line}

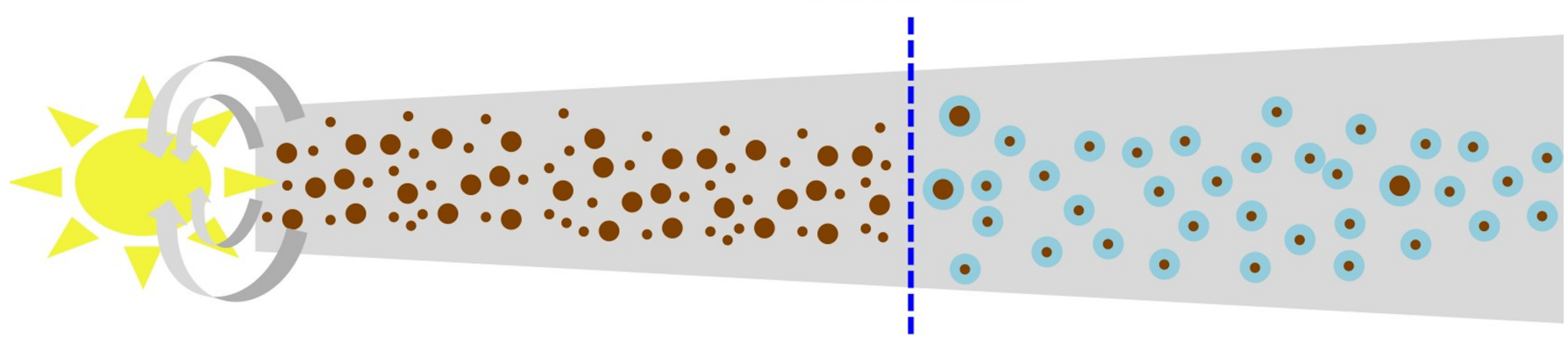

Extended Data Figure 1 | Sketch of the observed phenomenon. a, During quiescence, the water snow-line around stars of Solar masses is located $5 \mathrm{AU}$ or less from the star, where the temperature of the disk reaches the sublimation point of water. $\mathbf{b}$, During protostellar accretion outbursts, this line moves out to more than $40 \mathrm{AU}$, where it can be detected. Outward of the snow-line, grain growth is promoted by the high coagulation efficiency of ice-covered grains (brown and blue concentric circles). Inward of this line, dust production is promoted by the high fragmentation efficiency of bared silicates (brown circles). This results in the observed break in the disk intensity profile, a steep reduction in the $1.3-\mathrm{mm}$ dust opacity, and a sharp increase in the spectral index across the snow-line. 